\title{
Atribuciones causales de heteroagresión y autoagresión en jóvenes universitarios: Un análisis por sexo
}

\section{Heteroaggression and self-harm causal attributions in young university students: An analysis by sex}

\author{
José Luis Valdez Medina† \\ María Itzel Galván González \\ Nancy Neftalí Serrano Capula \\ Martha Adelina Torres Muñoz \\ Jorge Alvarado Orozco \\ Norma Ivonne González Arratia López Fuentes \\ Yessica Paola Aguilar Montes de Oca \\ Universidad Autónoma del Estado de México
}

\section{Resumen}

La presente investigación tuvo por objetivo identificar las conductas de heteroagresión y autoagresión más frecuentes en jóvenes universitarios. Para ello, se trabajó con 250 estudiantes de licenciatura de entre 18 y 25 años de edad, elegidos a través de un muestreo no probabilístico de tipo intencional. Distribuidos equitativamente por sexos, se les aplicó el cuestionario de conductas agresivas y autoagresivas de Valdez-Medina (2015), gracias al cual se determinó que $66 \%$ de los participantes ha llevado a la práctica la heteroagresión, pricipalmente debido a: la autodefensa, el desagrado, y la intolerancia. Del mismo modo, pudo observarse que 59.6\% de los encuestados declaró haberse autoagredido en algún momento de su vida, esto al sentirse frustrados, enojados y estresados. Ambos géneros llevaron a cabo dichos actos por la vía física, verbal y psicológica, lo que les permitió liberar su enojo y conseguir tranquilidad y alivio. Tales resultados, permitieron concluir que la experimentación de las emociones negativas de odio y frustración, se asocian con la presencia de conductas heteroagresivas y auto-agresivas, tanto en hombres como mujeres. Asimismo, se dedujo que la agresión y autoagresión efectuadas por los participantes fueron de corte adaptativo, ya que sus conductas buscaban preservar o recuperar el estado de paz o equilibrio perdido.

Palabras clave: heteroagresión, autoagresión, emociones negativas, jóvenes.

Nota del autor

José Luis Valdez Medina†; María Itzel Galván González, Facultad de Ciencias de la Conducta, Universidad Autónoma del Estado de México (UAEM); Nancy Neftalí Serrano Capula, Facultad de Ciencias de la Conducta, UAEM; Martha Adelina Torres Muñoz, Facultad de Ciencias de la Conducta, UAEM; Jorge Alvarado Orozco, Facultad de Ciencias de la Conducta, UAEM; Norma Ivonne González Arratia López Fuentes, Facultad de Ciencias de la Conducta, UAEM; Yessica Paola Aguilar Montes de Oca, Facultad de Ciencias de la Conducta, UAEM.

La correspondencia en relación con este artículo debe dirigirse a Norma Ivonne González Arratia López Fuentes, Facultad de Ciencias de la Conducta, UAEM, Filiberto Gómez S/N, Km. 1.5 TolucaNaucalpan, Col. Guadalupe, C.P. 50010, Toluca, Edo. de México.

Dirección electrónica: nigalf@yahoo.com.mx 


\begin{abstract}
This research aims to identify behaviors heteroaggression and more frequent self-harm in young university students. To do this, we worked with 250 students between 18 and 25 years old, chosen through a non probabilistic intentional sampling. To whom were administered the questionnaire aggressive and self-aggressive Valdez-Medina (2015) which showed that $66 \%$ of participants have implemented the heteroaggression, the main reasons for this action: self-defense, displeasure, and intolerance towards the other person. Similarly, it was observed that $59.6 \%$ of respondents have selfdeclared assaulted at some point in their life, that to feel frustrated, angry and stressed. They are noting that these acts were carried out in both sexes by the way: physical, verbal and psychological, which allowed them to release their anger and achieve tranquility and relief. These results led to the conclusion that experimentation of negative emotions of hate and frustration, are associated with the presence of hetero-aggressive behavior and self, both men and women. And that aggression and selfinjury was made by the participants adaptive court, as their behavior, seeking to preserve or restore the state of peace or lost balance.
\end{abstract}

Keywords: aggression, autoaggression, negative emotions, youth.

El Instituto Nacional de Estadística y Geografía (INEGI, 2015) reveló que en el 2014, se registraron 19 mil 669 homicidios en el país, los cuales tuvieron como principal causa la agresión con arma de fuego, seguida por los ataques con objetos no especificados y los llevados a cabo con objetos cortantes. En similar orden de ideas, el INEGI informó que en el 2012, 39, 826, 384 mujeres reportaron ser víctimas de violencia por parte de su última pareja, con mayor frecuencia de agresión emocional, seguida por la económica, la física y la sexual.

En similar orden de ideas, Villarroel et al. (2013) refieren que otra modalidad en la que suele presentarse la violencia, es la autoagresión. Y tal hecho es más común de lo esperado, pues estudios comunitarios aplicados a la población general, muestran que hasta $29 \%$ de los adolescentes y $6 \%$ de los adultos han presentado esta conducta al menos una vez en la vida. Mientras que la prevalencia de tales actos aumenta en la población con atención psiquiátrica, pues hasta $40 \%$ de los adolescentes y $21 \%$ de los adultos revelaron haber llevado a cabo alguna conducta autolesiva.

Ante tales cifras, Valdez-Medina (2015) considera necesario implementar acciones que en un primer momento ayuden a entender el fenómeno en cuestión, propone para ello ir al origen de la violencia, la cual de acuerdo con el autor, se hallaría en la agresión. Al respecto, Johnson (1976) y Berkowitz (1996) apuntan que la agresión es un tópico que se ha abordado desde hace décadas, aunque resulta complicado hacer un consenso sobre ¿qué es? Aun así, Chaux (2003) comenta que a grosso modo, la agresión puede ser entendida como el acto que tiene la intención de hacer daño a otro 
individuo. Acorde con Buss (1961, citado en Yudofsky, Silver, Jackson, Endicott \& Williams, 1986), la agresión tiene distintas expresiones, por ejemplo, Berkowitz (1996) habla de una dimensión verbal, cuando se emplea el lenguaje para infringir daño a otros.

Por su parte, Crick y Grotpeter (1995, citados en Gómez-Garibello \& Chaux, 2014) clasifican la agresión en física, cuando la intención es dañar corporalmente a una persona o a sus bienes. Por su lado, Baron y Richardson (1994) catalogan la agresión como directa, cuando se ejerce una conducta dañina contra quien es visto como el causante de una afección o afrenta. En el mismo orden, Richardson y Green (2003) hacen referencia a la agresión indirecta, descrita como cualquier acción encaminada a producir daño, siempre y cuando ésta se realice a través de otro individuo, objeto o pertenencia. En cambio, Goldstein y Keller (1991) hacen alusión a la agresión simbólica, consistente en degradar los emblemas apreciados por un grupo social. En el mismo sentido, Chaux (2003) hace referencia a la agresión relacional, reactiva e instrumental. La primera, implica dañar los vínculos sociales de la otra persona o al estatus social que tiene en su grupo, mientras que la agresión reactiva es el uso de la fuerza como respuesta a una ofensa real o percibida, en tanto que la agresión instrumental, es aquella que no está precedida de ningún agravio es empleada para conseguir objetivos. A la anterior categorización, Álvarez (2012) y Villarroel et al. (2013) anexan a la autoagresión, es decir, autoinfligirse, por voluntad propia, lesiones físicas, daño psicológico, desvalorización social, deterioro en la salud, entre otras acciones.

Una vez mencionadas algunas de las formas en que la agresión puede expresarse, es necesario hablar sobre de qué origina esta conducta. Al respecto, Johnson (1976) y Renfrew (2001) señalan que cualquier tipo de agresión es multicausal, pues el origen de éste puede ser genético -síndrome de Lesch-Nyhan, mutación del cromosoma $\mathrm{X}$ heredado por la madre-, hormonal -altos niveles de testosterona y estradiol-, neurológico -sobre-estimulación o daño del sistema límbico y tallo cerebral-, ambiental -exceso de ruido, aislamiento, aumento de la temperatura atmosférica- y psicológico -aprendizaje, imitación, emociones negativas, relaciones sociales inequitativas etc.-. En el presente estudio se hace hincapié en el conocimiento de las causales psicosocioculturales que llevan a los individuos a agredir a otros, o bien, a autoagredirse.

En otro orden de ideas, Berkowitz (1996) indica que las razones por las cuales una persona agrede a otra son varias, aun así éstas pueden clasificarse según su objetivo: coerción, cuando se busca influir sobre los otros para evitar que éstos realicen determinadas acciones; poder y dominio, cuando se pretende la conservación o fortalecimiento de la superioridad que se tiene sobre el atacado; y manejo de impresión, ya que al agredir se intenta dar una imagen de fortale- 
za ante los demás. Por su parte la autoagresión conforme a Battegay (1981) persigue objetivos un tanto distintos, ya que la persona que se autoinflige daño busca afecto, conservación, provocar conmoción en quienes lo rodean, superar sus ambivalencias y ser valorado como fuerte. A este comentario, se suma el de Álvarez (2012), quien postula que la autoagresión también tiene un fin catártico, aun y cuando es posible que cause más daños que beneficios.

Ahora bien, Pick (2000), Lloyd-Richardson et al. (2007), Papalia, Wendkos y Duskin (2009) y Archer, Fernández-Fuertes y Thanzami (2010) explican que las manifestaciones agresivas pueden aparecer en cualquier etapa de la vida. Aunque de acuerdo a Craig y Baucum (2001), durante la juventud aumenta la predisposición a caer en dicha conducta, debido al rol activo por desempeñar en cuatro contextos independientes, pero relacionados entre sí: individual, familiar, laboral-escolar, y sociocultural. Son justo esa exigencia e interacción de roles y ámbitos, las que según Corral (2009) crean una serie de dificultades con la pareja, familia, amigos, compañeros de escuela o trabajo y desconocidos, la cual puede desembocar en conatos o acciones agresivas. Por ello, en el presente trabajo se decidió especificarse en población de jóvenes, y en especial, aquellos que estuvieran cursando estudios de licenciatura, ya que para Craig y Baucum (2001), éstos son una población vulnerable, debido a las tensiones provocadas por las expectativas que se tiene depositadas en ellos.
Así pues, con el fin de contribuir al conocimiento con respecto a la forma en que los jóvenes universitarios viven la agresión y la autoagresión, se planteó la presente investigación con los siguientes objetivos: 1) identificar las conductas de heteroagresión y de autoagresión más frecuentes en jóvenes universitarios, 2) describir sus atribuciones causales, y 3) comparar la frecuencia y atribución causal entre ambos sexos.

\section{Método}

\section{Diseño de la investigación}

Se efectuó un estudio no experimental, de tipo exploratorio.

\section{Participantes}

Se trabajó con una muestra no probabilística de tipo intencional, integrada por 250 estudiantes universitarios -repartidos equitativamente por sexo y carrera- de las licenciaturas en Psicología, Trabajo Social, Educación, Derecho y Administración, que se imparten en la Universidad Autónoma del Estado de México (UAEM). La edad de los participantes osciló entre los 18 y 25 años, con una media de 23.5 años $(D E=0.90)$.

\section{Instrumento}

Se empleó el cuestionario de conductas agresivas y autoagresivas de Valdez-Medina (2015), el cual se compone de cinco preguntas 
abiertas, cuya validez fue obtenida a través de un jueceo por expertos, con un acuerdo mayor al $85 \%$.

Las preguntas que integran dicho cuestionario son: 1) ¿has agredido a alguien?,2) ¿Qué causó tu agresión?, 3) ¿te has autoagredido?, 4) ¿de qué forma te autoagrediste?, 5) ¿qué fue lo que te llevó a hacerlo?, y 6) ¿qué conseguiste o evitaste al autoagredirte?

\section{Procedimiento}

Una vez que los participantes firmaron el consentimiento informado, individualmente y en una sesión de 10 minutos, respondieron el cuestionario de conductas agresivas y autoagresivas de Valdez-Medina (2015), en un cubículo especial, dentro de las instalaciones de la UAEM.

\section{Análisis de datos}

Efectuadas las aplicaciones correspondientes, las respuestas obtenidas en cada uno los cuestionarios, fueron analizadas a través de la técnica de Análisis de Contenido, bajo la regla de numeración de frecuencia. Conforme a Bardin (1986) y Krippendorff (1997), esta última consiste en dividir el texto en categorías y subcategorías de análisis, para después anotar un símbolo cada que las unidades elegidas aparezcan. En un primer momento, se analizaron las respuestas conseguidas, para determinar las categorías y subcategorías de análisis propias de esta investigación. En una segunda etapa, los datos fueron analizados nuevamente y categorizados, así fueron representados por un símbolo cada que esa unidad aparecía.

\section{Resultados}

Tras la aplicación del cuestionario de conductas agresivas y autoagresivas de Valdez-Medina (2015), se procedió a realizar el análisis de contenido, cuyos resultados indican que $66 \%$ de los participantes ha agredido a otro individuo, acto que se acentuó en los hombres (ver tabla 1).

En el mismo orden de ideas, se registraron razones diversas por las cuales los varones agredieron a otra persona, a saber, sentirse violentado por ella, para protegerse, por enojo, porque un individuo les desagradó, por desesperación y porque en ese momento se les hizo divertido. Las féminas, por su parte,

\section{Tabla 1}

Comparación de las frecuencias y porcentajes de la pregunta cerrada ¿Has agredido a alguien?

\begin{tabular}{|c|c|c|c|c|c|c|c|c|c|c|c|}
\hline \multicolumn{4}{|c|}{ Hombres } & \multicolumn{4}{|c|}{ Mujeres } & \multicolumn{4}{|c|}{ Total } \\
\hline Sí & $\%$ & No & $\%$ & Sí & $\%$ & No & $\%$ & Sí & $\%$ & No & $\%$ \\
\hline 83 & 66.24 & 42 & 33.6 & 82 & 65.6 & 43 & 34.4 & 165 & 66 & 85 & 34 \\
\hline
\end{tabular}


comentaron haber agredido a alguien más debido a: sentirse forzada por ella, porque ésta les desagradaba, porque estaban enojadas, por desesperación, porque ya no toleraban a esa persona, por coraje y por estrés (ver tabla 2).
El tercer reactivo del cuestionario administrado, dejó ver que $59.6 \%$ de los participantes se ha autoagredido en algún momento de su vida (ver tabla 3).

Tabla 2

Comparación de las frecuencias y porcentajes de las categorías temáticas de la pregunta abierta ¿Qué causó tu agresión?

\begin{tabular}{|c|c|c|c|c|c|c|}
\hline \multirow{2}{*}{ Categoría } & \multicolumn{3}{|l|}{ Hombres } & \multicolumn{3}{|l|}{ Mujeres } \\
\hline & Subcategoría & $f$ & $\%$ & Subcategoría & $f$ & $\%$ \\
\hline Defensa & $\begin{array}{l}\text { Porque me agreden, ce- } \\
\text { los, agresión hacia seres } \\
\text { queridos, por problemas, } \\
\text { por hacerme perder un } \\
\text { objetivo }\end{array}$ & 15 & 32.50 & $\begin{array}{l}\text { Porque me agreden, acoso, } \\
\text { cuando me gritan, cuando } \\
\text { son injustos, agresión hacia } \\
\text { seres queridos, por maltrato } \\
\text { animal }\end{array}$ & 20 & 24.69 \\
\hline Intolerancia & $\begin{array}{l}\text { Desagrado, } \\
\text { desesperación, por gente } \\
\text { molesta, forma en que se } \\
\text { expresan, incompetencia } \\
\text { de los demás }\end{array}$ & 11 & 27.50 & $\begin{array}{l}\text { Desagrado, diferentes } \\
\text { ideologías, por comentarios } \\
\text { inadecuados, por su } \\
\text { forma de relacionarse, por } \\
\text { temperamental }\end{array}$ & 28 & 34.56 \\
\hline $\begin{array}{l}\text { Emoción } \\
\text { negativa }\end{array}$ & $\begin{array}{l}\text { Enojo, coraje, rencor, odio, } \\
\text { furia, ira }\end{array}$ & 12 & 30 & $\begin{array}{l}\text { Enojo, desesperación, coraje, } \\
\text { estrés, frustración, impotencia }\end{array}$ & 33 & 40.74 \\
\hline Diversión & $\begin{array}{l}\text { Borracheras, entre amigos, } \\
\text { placer, interacción en un } \\
\text { juego }\end{array}$ & 4 & 10 & ----- & 0 & 0 \\
\hline
\end{tabular}

Tabla 3

Comparación de las frecuencias y porcentajes de la pregunta cerrada ¿Te has autoagredido?

\begin{tabular}{|c|c|c|c|c|c|c|c|c|c|c|c|}
\hline \multicolumn{4}{|c|}{ Hombres } & \multicolumn{4}{|c|}{ Mujeres } & \multicolumn{4}{|c|}{ Total } \\
\hline Sí & $\%$ & No & $\%$ & Sí & $\%$ & No & $\%$ & Sí & $\%$ & No & $\%$ \\
\hline 64 & 51.2 & 61 & 48.8 & 85 & 68 & 40 & 32 & 149 & 59.6 & 101 & 40.4 \\
\hline
\end{tabular}


La cuarta interrogante del cuestionario de agresión y autoagresión de Valdez-Medina (2015), registró las causas que llevaron a los hombres a autoinfligirse daño: insulto, golpeándose contra la pared y sus manos, fumando, bebiendo alcohol, y saboteándose.
Las mujeres, por su parte, lo hicieron mediante el insulto, la desvalorización, cortándose, haciéndose sentir mal, consumiendo drogas, teniendo una baja autoestima y ofendiéndose a sí mismas (ver tabla 4).

Tabla 4

Comparación de las frecuencias y porcentajes, de las categorías temáticas de la pregunta abierta ¿De qué forma te autoagrediste?

\begin{tabular}{|c|c|c|c|c|c|c|}
\hline \multirow{2}{*}{ Categoría } & \multicolumn{3}{|l|}{ Hombres } & \multicolumn{2}{|l|}{ Mujeres } & \multirow[b]{2}{*}{$\%$} \\
\hline & Subcategorías & $f$ & $\%$ & Subcategorías & $f$ & \\
\hline Agresión verbal & Insultos, grosería & 12 & 18.75 & $\begin{array}{l}\text { Insultos, groserías, } \\
\text { regañarse a sí misma }\end{array}$ & 32 & 37.64 \\
\hline Agresión física & $\begin{array}{l}\text { Golpearse en la } \\
\text { pared, golpearse } \\
\text { manualmente, } \\
\text { tatuarse, exceso de } \\
\text { ejercicio }\end{array}$ & 16 & 25 & $\begin{array}{l}\text { Cortándose, ocasionando } \\
\text { peleas, dañarse la piel }\end{array}$ & 14 & 16.47 \\
\hline $\begin{array}{l}\text { Agresión } \\
\text { psicológica }\end{array}$ & $\begin{array}{l}\text { Pensar en algo que } \\
\text { lo afecta, emocional- } \\
\text { mente, pensarse } \\
\text { incapaz, pensar nega- } \\
\text { tivamente }\end{array}$ & 12 & 18.75 & $\begin{array}{l}\text { Desvalorizarse, } \\
\text { haciéndose sentir } \\
\text { mal, no aceptándose, } \\
\text { auto-sabotaje, pensar } \\
\text { negativamente, } \\
\text { emocionalmente, } \\
\text { recriminándose }\end{array}$ & 27 & 31.76 \\
\hline $\begin{array}{l}\text { Consumo de } \\
\text { estupefacientes }\end{array}$ & $\begin{array}{l}\text { Fumar, beber } \\
\text { alcohol, caer en vicios }\end{array}$ & 11 & 17.18 & $\begin{array}{l}\text { Drogándose, fumar, } \\
\text { beber alcohol }\end{array}$ & 4 & 4.70 \\
\hline Baja autoestima & $\begin{array}{l}\text { Menospreciarse, hacer } \\
\text { cosas que no quiere, } \\
\text { no siendo como es }\end{array}$ & 8 & 12.5 & Baja autoestima, moral & 3 & 3.52 \\
\hline Conductas & No hacer algo bien & 3 & 4.68 & $\begin{array}{l}\text { Perder oportunidades, } \\
\text { complacer al exnovio, } \\
\text { preocuparse por los } \\
\text { demás }\end{array}$ & 3 & 3.52 \\
\hline $\begin{array}{l}\text { Problemas } \\
\text { alimenticios }\end{array}$ & Dejar de comer & 1 & 1.56 & $\begin{array}{l}\text { Dejar de comer, comer } \\
\text { en exceso }\end{array}$ & 2 & 2.35 \\
\hline $\begin{array}{l}\text { Descuido de la } \\
\text { salud }\end{array}$ & Descuidar su salud & 1 & 1.56 & ----- & 0 & 0 \\
\hline
\end{tabular}


Asimismo, las causas que indujeron a los varones a autoagredirse fueron varias: el enojo, sentir coraje, los prejuicios, las presiones de su contexto, cometer errores, los fracasos, el gusto, y la curiosidad. Las féminas revelaron haberse autoinfligido daño por enojo, frustración, ser rechazada por los demás, la presión social, los errores cometidos, los fracasos vividos, baja autoestima, y como medio para liberar sus sentimientos (ver tabla 5).

Tabla 5

Comparación de las frecuencias y porcentajes, de las categorías temáticas de la pregunta abierta ¿Qué fue lo que te llevó a hacerlo?

\begin{tabular}{|c|c|c|c|c|c|c|}
\hline \multirow{2}{*}{ Categoría } & \multicolumn{3}{|l|}{ Hombres } & \multicolumn{2}{|l|}{ Mujeres } & \multirow[b]{2}{*}{$\%$} \\
\hline & Subcategoría & $f$ & $\%$ & Subcategoría & $f$ & \\
\hline $\begin{array}{l}\text { Emociones } \\
\text { negativas }\end{array}$ & $\begin{array}{l}\text { Enojo, coraje, depresión, } \\
\text { frustración, dolor, estrés, } \\
\text { soledad, impotencia, } \\
\text { tristeza, ansiedad, ira }\end{array}$ & 30 & 38.46 & $\begin{array}{l}\text { Enojo, frustración, } \\
\text { desesperación, estrés, } \\
\text { inseguridad, tristeza, } \\
\text { coraje, impotencia, } \\
\text { indecisión, ansiedad, dolor, } \\
\text { odio, depresión, soledad, } \\
\text { fracaso, presión }\end{array}$ & 41 & 48.23 \\
\hline $\begin{array}{l}\text { Aspectos } \\
\text { sociales }\end{array}$ & $\begin{array}{l}\text { Prejuicios, contexto, } \\
\text { presión social, imitación, } \\
\text { comportamientos de } \\
\text { otros }\end{array}$ & 11 & 14.10 & $\begin{array}{l}\text { Rechazo de los demás, } \\
\text { comportamientos de } \\
\text { otros, presión social }\end{array}$ & 3 & 3.52 \\
\hline $\begin{array}{l}\text { Actos } \\
\text { fallidos }\end{array}$ & $\begin{array}{l}\text { Cometer errores, fracaso, } \\
\text { inmadurez, corregir errores }\end{array}$ & 18 & 23.07 & $\begin{array}{l}\text { Cometer errores, fracaso, } \\
\text { olvidos }\end{array}$ & 21 & 24.70 \\
\hline Por placer & $\begin{array}{l}\text { Gusto, curiosidad, diver- } \\
\text { sión }\end{array}$ & 9 & 11.53 & $\begin{array}{l}\text { Liberar sentimientos, } \\
\text { curiosidad, diversión }\end{array}$ & 5 & 5.88 \\
\hline $\begin{array}{l}\text { Auto- } \\
\text { percepción }\end{array}$ & $\begin{array}{l}\text { Verme gordo, falta de } \\
\text { aceptación, no superar las } \\
\text { cosas, poco respeto hacia } \\
\text { mí }\end{array}$ & 4 & 5.12 & $\begin{array}{l}\text { Falta de aceptación, } \\
\text { sentirse mal }\end{array}$ & 4 & 4.70 \\
\hline $\begin{array}{l}\text { Baja } \\
\text { autoestima }\end{array}$ & Baja autoestima & 3 & 3.84 & $\begin{array}{l}\text { Baja autoestima, decep- } \\
\text { ción de sí mismos, falta de } \\
\text { confianza }\end{array}$ & 6 & 7.05 \\
\hline $\begin{array}{l}\text { Aspectos } \\
\text { personales }\end{array}$ & $\begin{array}{l}\text { Acciones personales, me- } \\
\text { jorar aspectos de mi vida }\end{array}$ & 3 & 3.84 & $\begin{array}{l}\text { Bajo control de impulsos, } \\
\text { mala decisión, necesidad } \\
\text { de compensar una falta }\end{array}$ & 5 & 5.88 \\
\hline
\end{tabular}


Gracias a la sexta pregunta, se detectó que ambos sexos obtuvieron ganancias al autoagredirse. En específico, los hombres expresaron haber conseguido placer, alivio, tranquilidad, tristeza, enojo, hacer lo pretendido, un amigo, tatuarse, experiencia, y problemas. En tanto que las mujeres reportaron haber logrado tranquilidad, desahogo, alivio, placer, comprensión, lograr una meta, corregir errores, trabajar sus emociones, bajar de peso, y problemas de salud (ver tabla 6).

Tabla 6.

Comparación de las frecuencias y porcentajes, de las categorías temáticas de la pregunta abierta ¿Qué conseguiste o evitaste al auto-agredirte?

\begin{tabular}{|c|c|c|c|c|c|c|}
\hline \multirow[b]{2}{*}{ Categoría } & \multicolumn{3}{|l|}{ Hombres } & \multicolumn{3}{|l|}{ Mujeres } \\
\hline & Subcategoría & $f$ & $\%$ & Subcategoría & $f$ & $\%$ \\
\hline $\begin{array}{l}\text { Emociones } \\
\text { positivas }\end{array}$ & $\begin{array}{l}\text { Placer, alivio, des- } \\
\text { estrés, tranquilidad, } \\
\text { paz, motivación, liberar } \\
\text { enojo, superación }\end{array}$ & 13 & 48.14 & $\begin{array}{l}\text { Tranquilidad, desahogarse, } \\
\text { liberar enojo, alivio, impulsarse, } \\
\text { respetarse, liberar endorfina, } \\
\text { placer, divertirse, salir de un } \\
\text { problema, ver lo bueno de su } \\
\text { error, solucionar su problemática }\end{array}$ & 18 & 62.02 \\
\hline $\begin{array}{l}\text { Emociones } \\
\text { negativas }\end{array}$ & $\begin{array}{l}\text { Tristeza, enojo, } \\
\text { agredirme }\end{array}$ & 4 & 14.81 & $\begin{array}{l}\text { Compensación, más preocupa- } \\
\text { ciones }\end{array}$ & 2 & 6.89 \\
\hline $\begin{array}{l}\text { Aspectos } \\
\text { personales }\end{array}$ & $\begin{array}{l}\text { Verme como quería, } \\
\text { ser más cuidadoso, } \\
\text { un tatuaje, hacer lo } \\
\text { que quería, amigos, } \\
\text { recordar lo que tengo } \\
\text { que hacer, experiencia }\end{array}$ & 7 & 25.92 & $\begin{array}{l}\text { Trabajar el manejo de emociones, } \\
\text { darse cuenta que estaba en un } \\
\text { error, bajar de peso, lograr una } \\
\text { meta }\end{array}$ & 6 & 27.58 \\
\hline $\begin{array}{l}\text { Problemas } \\
\text { físicos }\end{array}$ & $\begin{array}{l}\text { Estrés, dolor de } \\
\text { corazón }\end{array}$ & 3 & 11.11 & Dañar su salud & 1 & 3.44 \\
\hline
\end{tabular}

\section{Discusión}

Pick (2000); Lloyd-Richardson, Perrine, Dierker y Kelley (2007); así como Papalia, Wendkos y Duskin (2009), concuerdan que durante la juventud, los individuos son proclives a tener manifestaciones agresivas, por las nuevas exigencias a las cuales tienen que hacer frente de forma activa. Dicho postulado ha sido confirmado en la presente investigación, ya que $66 \%$ de los participantes manifestó haber agredido a otra persona. La razón principal que llevó a ambos sexos a incurrir en dicha 
conducta fue la experimentación de emociones negativas, en específico, enojo, coraje y rencor en los varones, así como enojo, desesperación y frustración en las féminas. Tal hallazgo permite documentar que las emociones negativas de odio y frustración, están asociadas con la agresión. Conocimiento que se suma a los datos empíricos previos, arrojados por los trabajos de Torres (2013) y Alvarado-Orozco (2014), quienes respectivamente, hallaron que las emociones negativas no resueltas se asocian con la presencia de síntomas somáticos, así como niveles de ansiedad y manifestaciones depresivas.

En el mismo sentido, pudo notarse que la segunda causa que llevó a ambos sexos a agredir a otra persona fue la defensa propia, ya fuera porque los participantes se hubieran sentido ofendidos o porque consideraron que alguno de sus familiares estaba siendo violentado. Tal resultado permite aseverar que los encuestados ejercieron primordialmente una agresión reactiva, la cual, según Chaux (2003) consiste en atacar a otro individuo, cuando se considera que éste ha cometido una ofensa real o imaginaria. Asimismo, es necesario mencionar que las respuestas obtenidas mostraron que, de acuerdo a Leal, Meneses, Alarcón y Karmelic (2005), así como de Valdez-Medina y Bastida (2014), los participantes ejercieron una agresión adaptativa, con la intención de estar fuera de peligro y sobrevivir.
Referente a la autoagresión, 59.6\% de los encuestados dijo haber efectuado esta conducta en algún momento; necesario es indicar que en comparación con los hombres, tal acto fue más frecuente en las mujeres. De igual forma, pudo advertirse que hubo diferencias por sexo al cometerse el daño, pues los varones optaron principalmente por el castigo físico, mientras que las féminas, eligieron primordialmente la autoagresión verbal y psicológica. Tal resultado conforme a Valdez-Medina et al. (2006), además de Archer et al. (2010) se debería a que de forma natural, los hombres tienden a manifestarse a través de las expresiones motor-gruesas, mientras que las mujeres, lo hacen mediante acciones que involucran la parte motor fina y la palabra.

Al igual que ocurrió con la heteroagresión, la experimentación de frustración y odio se asoció con la presencia de autoagresión, lo que constituye otra evidencia empírica con respecto a las repercusiones que la vivencia de emociones negativas trae para las personas. Asimismo, fue de llamar la atención que la segunda causa que llevó a los participantes a autoinfligirse daño fue el cometer errores y tener fracasos. Tal suceso encontraría explicación en lo formulado por Goldstein y Keller (1991) y Lloyd-Richardson et al. (2007), según quienes las personas que se autoagreden, lo hacen por impulsividad y una baja tolerancia a la frustración, esto desemboca en un mal manejo de las contingencias, donde 
la única posibilidad aparente para solventar su error es dañarse. En cambio, para ValdezMedina (2009) cuando un individuo se autoagrede, manifiesta culpa, y con el daño que se provoca, busca atenuar el castigo que pueda llegar a recibir por parte del que fue agraviado con sus acciones.

Ahora bien, en comparación con los varones, la autoagresión fue más frecuente en las féminas, acto que para Lagarde (2000) y Alazanes, Ortiz, Del Olmo y Troche (2005) es consecuencia de los reproches que las mujeres se hacen, debido a las exigencias de la nueva faceta en la que se han visto enroladas, además de cumplir con su papel tradicional de ama de casa, han de ser independientes y profesionistas exitosas.

De igual modo, pudo observarse que una vez infligido la autoagresión, ambos sexos dijeron sentirse tranquilos o aliviados, peculiaridad que se acentuó en las féminas. Para Silva y Gálviz (2010), Richardson y Green (2003) así como para Rigo (2013) dicha circunstancia se debe a que quienes se autoinfligen daño, lo hacen para obtener el control de una situación y liberar tensión emocional, o bien, porque el dolor físico los distrae de emociones y pensamientos aversivos. Sin embargo, aunque fueron la minoría, hubo mujeres y hombres (en mayor número) que reconocieron haber puesto en riesgo su salud física y emocional. Tal reflexión empata con lo manifestado por Martin (2000), para quien la autoagresión es un importante factor de riesgo para la vida y la salud, aun y cuando su práctica no tenga intención suicida.
Así pues, los datos recabados en la presente investigación permiten realizar las siguientes conclusiones.Primera.E166\%delosparticipantes ha agredido a otro individuo debido a la búsqueda de autoprotección, en el caso de los hombres, y por la vivencia de emociones negativas, en el caso de las mujeres. Segunda. El 59.6\% de los encuestados se ha autoagredido en el algún momento de su vida; en el caso de los varones tal daño fue administrado primordialmente de forma física, mientras que las mujeres dijeron violentarse principalmente de manera verbal, esto a raíz de la experimentación de emociones negativas. Después de haberse autoinfligido el daño -característica que se acentuó en las féminas-, ambos sexos reportaron la vivencia de alivio y placer. Tercera. Pudo documentarse que en ambos sexos, la experimentación de odio y frustración están asociadas con la presencia de conductas autoagresivas y heteroagresivas. Cuarta. Los hombres tienden a la heteroagresión, mientras que las féminas lo hacen a la autoagresión. Quinta. Con base en el análisis, se determinó que los encuestados llevaron a cabo, principalmente, agresión y autoagresión de tipo reactiva y adaptativa.

Al igual que ocurre con otros estudios, es necesario tomar con cautela las resultas y conclusiones del presente trabajo, ya que al ser un estudio con muestreo no probabilístico, de acuerdo a Rojas (1982) y Zorrilla (2001), la información recabada no puede representar a la población general. De igual modo, otra limitante que posee el presente estudio tiene relación con 
la población elegida, ya que el ser estudiante universitario implica haber cursado al menos 15 años de educación formal, lo que en teoría proporciona al individuo una mayor capacidad de discernimiento, ante sus problemas. Y eso, sin duda, influyó en los resultados obtenidos. Así mismo, el desequilibrio de la $\mathrm{N}$ en todas las subcategorías impidió realizar un análisis estadístico, y por ende, dar un sustento más robusto a las conclusiones efectuadas.

Se recomienda a quienes pretenden realizar un estudio similar al presente, incluyan en la muestra a jóvenes de ámbitos distintos al escolar. Además, se aconseja también indagar la frecuencia con que los participantes incurren en la conducta de heteroagresión y autoagresión, para así llevar el control sobre el número de respuestas dadas en cada pregunta, con el fin de utilizar pruebas estadísticas de la categorización.

\section{Referencias}

Alazanes, J., Ortiz, M., Del Olmo, D., \& Troche, P. (2005). Sociedad y familia. En H. Oudhof, M. J. Morales, \& P. Troche (Eds.), Memoria del foro "Familia y juventud" (pp. 21-23). Estado de México, México: Universidad Autónoma del Estado de México.

Alvarado-Orozco, J. (2014). Frustración, odio y culpa, origen de la personalidad en conflicto (Tesis de maestría inédita). Faculta de Ciencias de la Conducta, Universidad Autónoma del Estado de México, Toluca, Estado de México.
Álvarez (2012). Autoagresión, dañar el cuerpo para aliviar el alma. Recuperado de: http://www.autolesion.com/2012/08/24/ autoagresion-mutilar-el-cuerpo-para-aliviarel-alma/.

Archer, J.,Fernández-Fuertes,A.A., \& Thanzami, V. L. (julio, 2010). Does cost-benefit analysis or self-control predict involvement in two forms of aggression? Aggressive Behavior, 36. Recuperado de http://www.readcube. com/articles/10.1002\%2Fab.20358?r3 _ referer=wol\&tracking_action=preview click\&show_checkout=1\&purchase_ referrer=onlinelibrary. wiley. com\&purchase_site_license $=$ LICENSE_ DENIED.

Bardin, L. (1986). Análisis de Contenido. Madrid, España: Akal.

Baron, R. A., \& Richardson, D. R. (1994). Human Aggression. New York, EEUU: Plenum Press.

Battegay, R. (1981). La agresión. Barcelona, España: Editorial Herder.

Berkowitz, L. (1996). Agresión, consecuencias y control. Bilbao, España: Desclée De Brouwer.

Chaux, E. (2003). Agresión reactiva, agresión instrumental y el ciclo de la violencia. Revista de Estudios Sociales, 15. Recuperado de http://www.redalyc.org/articulo.oa?id= 81501504.

Corral, A. (2007). El desarrollo psicológico durante la vida adulta. En E. Begoña (Coord.), Psicología del desarrollo, desde la infancia 
a la vejez, Vol. 2 (pp. 167-184). Madrid, España: Editorial MacGraw-Hill.

Craig, G. J., \& Baucum, D. (2001). Desarrollo psicológico ( $8^{\mathrm{a}}$ ed.). Estado de México, México: Pearson Educación.

Goldstein, A. P., \& Keller, H. R. (1991). El comportamiento agresivo. Bilbao, España: Desclée de Brouwer.

Gómez-Garibello, C., \& Chaux, E. (2014). Agresión relacional en preescolar: Variables cognoscitivas y emocionales asociadas. Universitas Psychologica, 13(2). doi:10.11144/ Javeriana.UPSY13-2.arpv.

Instituto Nacional de Estadística y Geografía (2012). Violencia en los hogares. Recuperado dehttp://www3.inegi.org.mx/sistemas/sisept/ default.aspx $? \mathrm{t}=$ mvio46\&s $=$ est $\& \mathrm{c}=26520$

Instituto Nacional de Estadística y Geografía (2015). Datos preliminares de homicidios en 2014. Recuperado de http://www.beta.inegi. org.mx/temas/incidencia/

Johnson, R. N. (1976). La agresión en el hombre y animales. Distrito Federal, México: Manual Moderno.

Krippendorff, K. (1997). Metodología del análisis de contenido, teoría y práctica ( $1^{\mathrm{a}}$ reimp.). Barcelona, España: Paidós.

Lagarde, M. (2000). Los cautiverios de las mujeres; madre, esposa, monjas, putas, presas, y locas. Distrito Federal, México: Universidad Nacional Autónoma de México.

Leal, F., Meneses, M., Alarcón, A., \& Karmelic, V. (2005). Agresión y resentimiento en los es- tudiantes secundarios. Revista de Psicología, 14(1). Recuperado de http://www.redalyc. org/articulo.oa? $\mathrm{id}=26414103$.

Lloyd-Richardson, E., Perrine, N., Dierker, L., \& Kelley, M. (2007). Characteristics and functions of non-suicidal self-injury in a community sample of adolescents. Psychol Med, 37(8). Recuperado de http://www.ncbi. nlm.nih.gov/pmc/articles/PMC2538378/.

Martín, J. (2000). Agresión: un enfoque psicobiológico. Valencia, España: Promolibro.

Papalia, D., Wendkos, S., \& Duskin, R. (2009). Psicología del desarrollo: de la infancia a la adolescencia. Distrito Federal, México: McGraw Hill.

Pick, S. (2000). Yo, Adolescente: Respuestas Claras a mis Grandes Dudas. Distrito Federal, México: Planeta.

Renfrew, J. W. (2001). La agresión y sus causas. Distrito Federal, México: Editorial Trillas.

Richardson, D. S., \& Green, L. R. (2003). Defining direct and indirect aggression: The Richardson Conflict Response Questionnaire. Revue international de psychologie sociale, 16(3). Recuperado de http://cat.inist.fr/?aMo dele $=$ afficheN\&cpsidt $=15279315$

Rigo. E. (2013). Autolesiones ¿Por qué lo hacen?, todo lo que necesitas saber y cómo enfrentarte a ellas. Recuperado de: http://www. medciencia.com/autolesiones-porque-lohacen-todo-lo-que-necesitas-saber-y-comoenfrentarte-a-ellas-trigger/. 
Rojas, R. (1982). Guía para realizar investigaciones sociales ( $7^{\mathrm{a}}$ ed.). Distrito Federal, México: Universidad Nacional Autónoma de México.

Silva, A., \& Gálviz, L. (2010). Silencio y grito de la adolescencia. Fermentum. Revista Venezolana de Sociología y Antropología, 20(58). Recuperado de http://www.saber.ula. ve/bitstream/123456789/35204/1/articulo1. pdf.

Torres, M. A. (2013). Características de personalidad en adultos que presentan síntomas somáticos (Tesis de maestría inédita). Facultad de Ciencias de la Conducta, Universidad Autónoma del Estado de México, Toluca, Estado de México.

Valdez Medina, J. L., \& Bastida, R. (2014). Del instinto de defensa del territorio a la agresión y violencia, desde la teoría de la paz o equilibrio. Conferencia magistral presentada en Segunda Semana de Psicología Social. Facultad de Psicología, Universidad Nacional Autónoma de México, México, Distrito Federal, México.

Valdez-Medina, J. L. (2009). Teoría de la Paz o Equilibrio. México: EDAMEX.

Valdez-Medina, J. L. (2015). Estancia integrativa metodológica [Seminario]. Universidad Autónoma del Estado de México, Licenciatura en Psicología, Facultad de Ciencias de la Conducta. Toluca, Estado de México. 17 de marzo de 2015.
Valdez-Medina, J. L., Díaz-Loving, R., \& Pérez M. R. (2006). Los hombres y las mujeres en México: Dos mundos distantes y complementarios. Toluca, México: Universidad Autónoma del Estado de México.

Villarroel, J., Jerez, S., Montenegro, A., Montes, C., Igor, M., \& Silva, I. (2013). Conductas autolesivas no suicidas en la práctica clínica. Revista Chilena de Neuro-psiquiatría, 51(1). Recuperado de http://www.redalyc.org/articulo.oa?id=331527733006.

Yudofsky, S. C., Silver, M. J., Jakson, W., Endicott, J., \& Williams, D. (1986). The overt aggression scale for the objective rating of verbal and physical aggression. American Journal of Psychiatry, 143(1). Recuperado de http://www.researchgate.net/publication/19220225_The_Overt_Aggression_ Scale_for_the_Objective_Rating_of_Verbal_and_Physical_Aggression.

Zorrilla, S. (2001). Introducción a la metodología de la investigación (25 ed.). Distrito Federal, México: Aguilar León.

Recibido el 05 de octubre de 2015

Revisado el 12 de enero de 2016

Aceptado el 17 de febrero de 2016 